Annuaire suisse de politique de développement

$21 \mid 2002$

Agriculture suisse et mondialisation

\title{
4. Relations de la Suisse avec les pays en développement et en transition
}

Gérard Perroulaz, Antonio Girardi et Nicolas Roguet

\section{(2) OpenEdition}

1 Journals

Édition électronique

URL : http://journals.openedition.org/aspd/956

DOI : 10.4000/aspd.956

ISSN : 1663-9669

Éditeur

Institut de hautes études internationales et du développement

Édition imprimée

Date de publication : 1 avril 2002

Pagination : 359-364

ISSN : 1660-5934

\section{Référence électronique}

Gérard Perroulaz, Antonio Girardi et Nicolas Roguet, « 4. Relations de la Suisse avec les pays en

développement et en transition », Annuaire suisse de politique de développement [En ligne], 21 | 2002,

mis en ligne le 07 septembre 2012, consulté le 08 septembre 2020. URL : http://

journals.openedition.org/aspd/956 ; DOI : https://doi.org/10.4000/aspd.956 


\section{RELATIONS DE LA SUISSE AVEC LES PAYS EN DÉVELOPPEMENT ET EN TRANSITION*}

\subsection{Relations avec les pays en développement, 2000 , en milliers de francs}

\begin{tabular}{|c|c|c|c|c|c|c|}
\hline Continent/pays & IPD, aide bila & Aide priv. ${ }^{2}$ & $\mathbf{G R E}^{3}$ & $\mathrm{IDE}^{4}$ & Import. $^{5}$ & Export. $^{6}$ \\
\hline Total & 1058870,2 & 274146,0 & 844433,4 & 1927300,0 & 8995284,2 & 15589287,8 \\
\hline Europe & 154498,6 & 54305,0 & $-9689,6$ & $-230400,0$ & 645064,4 & 2111729,6 \\
\hline $\begin{array}{l}\text { Pays à faible revenu } \\
\text { (hors PMA) }\end{array}$ & 2992,4 & - & - & - & 7512,2 & 6982,5 \\
\hline Moldova & 2992,4 & - & - & - & 7512,2 & 6982,5 \\
\hline Pays à revenu moyen & 108573,5 & 51158,8 & $-6769,7$ & $-235200,0$ & 470612,3 & 1773975,1 \\
\hline Albanie & 11809,6 & 2439,1 & - & - & 461,7 & 9085,3 \\
\hline Bosnie-Herzégovine & 18945,9 & 4348,3 & - & - & 3218,3 & 32213,3 \\
\hline Croatie & 3257,4 & 540,6 & 322,1 & 6800,0 & 35966,9 & 169656,2 \\
\hline Macédoine (ERYM) & 15473,5 & 12,1 & - & - & 12882,1 & 38949,2 \\
\hline Turquie & 9832,6 & 5267,3 & $-7091,9$ & $-242000,0$ & 403232,4 & 1412408,7 \\
\hline Yougoslavie, Rép. féd. & 49254,4 & 38551,5 & - & - & 14850,9 & 111662,4 \\
\hline Pays à revenu élevé & 68,0 & 13,7 & $-2919,9$ & 2200,0 & 166939,9 & 330772,0 \\
\hline Malte & 68,0 & - & - & 5100,0 & 3826,4 & 71127,2 \\
\hline Slovénie & - & 13,7 & $-2919,9$ & $-2900,0$ & 163113,5 & 259644,8 \\
\hline $\begin{array}{l}\text { Non ventilés } \\
\text { et projets régionaux }\end{array}$ & 42864,6 & 3132,5 & - & 2600,0 & & \\
\hline $\begin{array}{l}\text { Etats Ex-Yougoslavie, } \\
\text { non spécifié }\end{array}$ & 36675,6 & 3066,4 & - & - & & \\
\hline Europe, non ventilé & 6189,0 & 66,1 & - & 2600,0 & & \\
\hline Afrique & 273257,7 & 81913,1 & 31122,0 & 119100,0 & 1084514,7 & 2048246,1 \\
\hline $\begin{array}{l}\text { Pays les moins avancés } \\
\text { (PMA) }\end{array}$ & 206376,7 & 59513,1 & $-4693,1$ & $-400,0$ & 50058,3 & 241141,8 \\
\hline Angola & 5891,3 & 1344,4 & $-4693,1$ & - & 6,3 & 7986,3 \\
\hline Bénin & 10129,0 & 1301,6 & - & - & 343,7 & 26417,8 \\
\hline Burkina Faso & 19363,5 & 2522,3 & - & - & 281,7 & 1129,5 \\
\hline Burundi & 5722,6 & 1066,7 & - & - & 4350,2 & 456,1 \\
\hline Cap Vert & 5096,1 & 82,0 & - & - & 3,4 & 472,1 \\
\hline Centrafricaine, Rép. & 142,4 & 233,2 & - & - & 1089,0 & 79,1 \\
\hline Comores & - & 86,3 & - & - & 120,2 & 17,5 \\
\hline
\end{tabular}

1. APD, aide bilat. $=$ aide publique au développement, aide bilatérale .

2. Aide priv. $=$ aide privée des ONG.

3. GRE = garantie contre les risques à l'exportation.

4. IDE = flux d'investissements directs à l'étranger.

5. Import. $=$ commerce, importations de la Suisse en provenance de...

6. Export. $=$ exportations suisses vers...

* Par Gérard Perroulaz, Antonio Girardi, Nicolas Roguet, service statistique de la DDC. 


\subsection{Relations avec les pays en développement, 2000, en milliers de francs (suite)}

$\begin{array}{llllll}\text { Continent/pays } & \text { APD, aide bilat. Aide priv. } & \text { GRE } & \text { IDE } & \text { Import. } & \text { Export. }\end{array}$

\begin{tabular}{|c|c|c|c|c|c|c|}
\hline Congo, Rép. Dém. & 6723,5 & 3750,6 & - & $-400,0$ & 2162,1 & 3463,1 \\
\hline Djibouti & 705,2 & 114,7 & - & - & 1,4 & 271,3 \\
\hline Erythrée & 2603,5 & 1242,5 & - & - & 4,8 & 650,5 \\
\hline Ethiopie & 6053,5 & 6485,2 & - & - & 6709,0 & 4964,7 \\
\hline Gambie & - & 127,8 & - & - & 5,7 & 933,7 \\
\hline Guinée & 1147,1 & 1087,1 & - & - & 237,6 & 13798,2 \\
\hline Guinée Equatoriale & 55,5 & 209,5 & - & - & 0,9 & 295,2 \\
\hline Guinée-Bissau & 4189,9 & 172,5 & - & - & 0,0 & 1900,4 \\
\hline Lesotho & 2057,4 & 519,0 & - & - & 26,1 & 10,7 \\
\hline Libéria & 607,3 & 112,1 & - & - & 3456,0 & 13556,5 \\
\hline Madagascar & 7606,7 & 2308,4 & - & - & 2631,0 & 3198,5 \\
\hline Malawi & - & 428,9 & - & - & 10779,3 & 660,5 \\
\hline Mali & 12888,0 & 2532,9 & - & - & 977,3 & 1528,3 \\
\hline Mauritanie & 199,8 & 1156,7 & - & - & 37,5 & 2208,0 \\
\hline Mozambique & 42351,2 & 8980,1 & - & - & 585,2 & 6069,4 \\
\hline Niger & 13074,9 & 451,3 & - & - & 295,6 & 94572,3 \\
\hline Ouganda & 2039,7 & 1708,2 & - & - & 7724,0 & 3023,7 \\
\hline Rwanda & 8503,1 & 2641,1 & - & - & 750,3 & 382,8 \\
\hline Sao Tomé \& Principe & - & - & - & - & 180,7 & 422,4 \\
\hline Sierra Leone & 4487,7 & 74,1 & - & - & 142,5 & 1338,6 \\
\hline Somalie & 2313,0 & 1161,6 & - & - & 165,3 & 132,9 \\
\hline Soudan & 9345,1 & 9894,5 & - & - & 394,1 & 31362,0 \\
\hline Tanzanie & 21376,9 & 4338,8 & - & - & 1195,2 & 7808,8 \\
\hline Tchad & 9858,4 & 2002,7 & - & - & 34,7 & 1261,6 \\
\hline Togo & 1093,8 & 690,7 & - & - & 4797,7 & 7983,6 \\
\hline Zambie & 750,7 & 685,6 & - & - & 569,8 & 2785,7 \\
\hline $\begin{array}{l}\text { Pays à faible revenu } \\
\text { (hors PMA) }\end{array}$ & 12675,5 & 11851,1 & $-885,0$ & $-14000,0$ & 110123,1 & 231759,9 \\
\hline Cameroun & 3487,2 & 4373,3 & $-93,2$ & - & 3832,7 & 11074,8 \\
\hline Congo, Rép. & 1006,3 & 144,6 & - & - & 2162,1 & 11260,1 \\
\hline Côte d'Ivoire & 523,1 & 343,0 & $-146,6$ & $-24500,0$ & 14188,6 & 35599,8 \\
\hline Ghana & 1109,5 & 1661,9 & - & - & 32469,9 & 20750,9 \\
\hline Kenya & 1399,7 & 1423,6 & $-645,3$ & 8000,0 & 18529,9 & 28980,1 \\
\hline Nigéria & 215,3 & 235,6 & - & 2500,0 & 295,6 & 94572,3 \\
\hline Sénégal & 4309,4 & 1670,0 & - & - & 5342,4 & 13061,8 \\
\hline Zimbabwe & 625,0 & 1999,1 & - & - & 33301,9 & 16460,1 \\
\hline Pays à revenu moyen & 26352,6 & 7158,1 & 36700,1 & 97500,0 & 924333,3 & 1575344,4 \\
\hline Afrique du Sud & 9033,4 & 1765,1 & $-4940,5$ & 1700,0 & 503994,8 & 604283,9 \\
\hline Algérie & 1645,6 & 603,4 & 42118,9 & - & 282274,7 & 129059,2 \\
\hline Botswana & - & 208,0 & - & - & 179,7 & 3709,8 \\
\hline Egypte & 11824,9 & 1572,3 & 1722,7 & 7200,0 & 12044,5 & 541262,0 \\
\hline Gabon & 4,0 & 950,3 & - & - & 512,2 & 2591,2 \\
\hline Maroc & 874,2 & 873,6 & $-1407,3$ & $-26000,0$ & 72733,7 & 133990,3 \\
\hline
\end{tabular}




\subsection{Relations avec les pays en développement, 2000 , en milliers de francs (suite)}

Continent/pays APD, aide bilat. Aide priv. GRE IDE Import. Export.

\begin{tabular}{|c|c|c|c|c|c|c|}
\hline Maurice & - & - & 894,2 & 97800,0 & 29822,8 & 49592,2 \\
\hline Namibie & 394,3 & 213,2 & - & - & 547,8 & 2109,3 \\
\hline Seychelles & - & - & - & - & 73,2 & 2174,9 \\
\hline Swaziland & - & 564,8 & - & - & 53,7 & 654,7 \\
\hline Tunisie & 2576,2 & 407,5 & $-1687,9$ & 16800,0 & 22096,2 & 105916,9 \\
\hline $\begin{array}{l}\text { Non ventilés } \\
\text { et projets régionaux }\end{array}$ & 27852,8 & 3390,8 & - & 36000,0 & & \\
\hline Afrique, non spécifié & 27852,8 & 3390,8 & - & 36000,0 & & \\
\hline Amérique & 107831,2 & 56987,6 & 377924,8 & 2302400,0 & 1625675,4 & 3818508,6 \\
\hline $\begin{array}{l}\text { Pays les moins avancés } \\
\text { (PMA) }\end{array}$ & 3952,5 & 4851,8 & - & - & 3192,5 & 2472,2 \\
\hline Haïti & 3952,5 & 4851,8 & - & - & 3192,5 & 2472,2 \\
\hline $\begin{array}{l}\text { Pays à faible revenu } \\
\text { (hors PMA) }\end{array}$ & 20705,0 & 12430,9 & - & 5500,0 & 29965,6 & 42218,6 \\
\hline Honduras & 9021,5 & 5646,0 & - & 5500,0 & 16170,1 & 34711,3 \\
\hline Nicaragua & 11683,5 & 6784,9 & - & - & 13795,5 & 7507,3 \\
\hline Pays à revenu moyen & 64467,9 & 38501,7 & 377924,8 & 2308600,0 & 1592517,3 & 3773817,8 \\
\hline Antigua \& Barbuda & - & - & - & - & 385,8 & 2426,1 \\
\hline Argentine & 329,4 & 525,1 & $-9947,0$ & 27600,0 & 76508,0 & 405296,6 \\
\hline Barbade & - & - & - & 42500,0 & 2307,5 & 3432,1 \\
\hline Bélize & 75,0 & - & - & - & 1716,2 & 343,0 \\
\hline Bolivie & 17802,2 & 4850,1 & 606,4 & 400,0 & 1453,1 & 10159,9 \\
\hline Brésil & 3057,2 & 7761,1 & 7631,3 & 156800,0 & 855661,6 & 1262042,7 \\
\hline Chili & 698,7 & 1310,7 & $-2413,0$ & $-43500,0$ & 80821,8 & 150397,5 \\
\hline Colombie & 9471,5 & 5677,6 & $-7001,5$ & $-98600,0$ & 79043,2 & 164228,9 \\
\hline Costa Rica & 1765,4 & 129,5 & - & $-17200,0$ & 60305,3 & 42812,2 \\
\hline Cuba & 1161,2 & 95,5 & - & - & 29917,3 & 6495,8 \\
\hline Dominicaine, Rép. & 1359,5 & 1235,7 & $-94,2$ & 99300,0 & 11119,5 & 21762,6 \\
\hline Dominique & 60,0 & - & - & - & 289,1 & 677,2 \\
\hline El Salvador & 4068,7 & 2415,0 & - & $-600,0$ & 6217,2 & 27594,7 \\
\hline Equateur & 7869,4 & 2168,0 & - & 19500,0 & 34854,4 & 43411,9 \\
\hline Granade & - & - & - & - & 935,7 & 613,0 \\
\hline Guatemala & 2607,8 & 4498,3 & $-3105,6$ & $-65000,0$ & 23459,3 & 28208,3 \\
\hline Guyana & - & - & - & - & 10,2 & 504,1 \\
\hline Jamaique & - & - & - & - & 5494,4 & 20244,1 \\
\hline Mexique & 513,4 & 933,9 & 360853,2 & 801100,0 & 191120,0 & 992576,6 \\
\hline Panama & 99,8 & 901,2 & $-262,6$ & 1039300,0 & 48051,2 & 269728,8 \\
\hline Paraguay & 1324,1 & 858,2 & - & - & 4316,0 & 19207,3 \\
\hline Pérou & 10850,7 & 3067,3 & 44,0 & 8200,0 & 21085,1 & 73761,8 \\
\hline Trinité \& Tobago & - & 8,5 & - & - & 646,5 & 6734,9 \\
\hline Uruguay & 240,6 & 76,9 & 297,5 & 274000,0 & 33389,3 & 74083,5 \\
\hline Vénézuela & 1113,1 & 1989,2 & 31316,5 & 64800,0 & 23409,6 & 147074,2 \\
\hline
\end{tabular}




\subsection{Relations avec les pays en développement, 2000, en milliers de francs (suite)}

Continent/pays APD, aide bilat. Aide priv. GRE IDE Import. Export.

\begin{tabular}{|c|c|c|c|c|c|c|}
\hline $\begin{array}{l}\text { Non ventilés } \\
\text { et projets régionaux }\end{array}$ & 18705,8 & 1203,2 & - & $-11700,0$ & & \\
\hline Amérique non spécifié & 18705,8 & 1203,2 & - & $-11700,0$ & & \\
\hline Asie & 229370,2 & 57215,6 & 445076,2 & $-257600,0$ & 5639680,8 & 7608755,1 \\
\hline $\begin{array}{l}\text { Pays les moins avancés } \\
\text { (PMA) }\end{array}$ & 65112,9 & 15550,4 & - & $-5200,0$ & 105782,1 & 121326,8 \\
\hline Afghanistan & 8629,7 & 3273,6 & - & - & 433,4 & 1742,2 \\
\hline Bangladesh & 24672,0 & 5031,6 & - & $-5600,0$ & 65944,5 & 79288,5 \\
\hline Bhoutan & 5742,0 & 801,8 & - & - & 36,0 & 428,8 \\
\hline Cambodge & 3112,5 & 2648,0 & - & 400,0 & 6451,3 & 3900,3 \\
\hline Laos & 2642,2 & 905,1 & - & - & 7529,4 & $656^{\prime} 2$ \\
\hline Myanmar (Birmanie) & 682,0 & 931,3 & - & - & 3666,8 & 2475,9 \\
\hline Népal & 19623,8 & 1958,9 & - & - & 21585,7 & 5649,5 \\
\hline Yémen & 8,9 & - & - & - & 135,0 & 27841,6 \\
\hline $\begin{array}{l}\text { Pays à faible revenu } \\
\text { (hors PMA) }\end{array}$ & 114037,3 & 28794,5 & $-170701,8$ & 126400,0 & 3843440,4 & 3698090,3 \\
\hline Arménie & 591,0 & 1189,5 & - & - & 1320,8 & 4679,0 \\
\hline Azerbaidjan & 1071,7 & - & 26819,9 & - & 1890,2 & 42899,9 \\
\hline Chine & 16702,6 & 1274,3 & $-1880,1$ & 198200,0 & 2300549,3 & 1402658,2 \\
\hline Corée, Dém. & 4410,4 & 536,0 & - & - & 532923,7 & 1058410,1 \\
\hline Inde & 30914,6 & 16832,8 & $-23530,8$ & $-38000,0$ & 600629,4 & 655673,9 \\
\hline Indonésie & 5898,6 & 2619,8 & $-163518,8$ & $-53100,0$ & 206873,1 & 215695,9 \\
\hline Kirghize, Rép. & 12214,7 & 421,6 & - & - & 99,3 & 800,1 \\
\hline Mongolie & 143,8 & 115,4 & - & - & 899,9 & 4774,0 \\
\hline Pakistan & 15020,2 & 501,1 & $-9886,0$ & 53300,0 & 47279,1 & 222547,6 \\
\hline Tadjikistan & 8498,7 & 691,0 & - & - & 682,3 & 867,2 \\
\hline Timor Oriental & 554,2 & 26,2 & - & - & 502,2 & 177,5 \\
\hline Turkménistan & - & - & - & - & 3348,2 & 10529,6 \\
\hline Vietnam & 18016,9 & 4586,9 & 1294,1 & $-34000,0$ & 146442,9 & 78377,3 \\
\hline Pays à revenu moyen & 37642,7 & 12122,6 & 615778,0 & $-359100,0$ & 1690458,3 & 3789338,0 \\
\hline Arabie Saoudite & - & - & $-24090,9$ & $-85100,0$ & 196622,4 & 986195,4 \\
\hline Bahrein & - & - & 980,3 & - & 19057,0 & 100389,5 \\
\hline Géorgie & 3863,0 & 24,2 & - & - & 116,5 & 5479,4 \\
\hline Irak & 6023,7 & 922,0 & - & - & 0,0 & 113604,7 \\
\hline Iran & 2413,2 & 155,8 & 245344,4 & 900,0 & 183173,3 & 393339,7 \\
\hline Jordanie & 6085,5 & 94,0 & $-1259,0$ & - & 753,8 & 90063,4 \\
\hline Kazakhstan & 601,4 & 649,6 & - & 50900,0 & 15941,2 & 25111,6 \\
\hline Liban & 795,3 & 1390,4 & - & $-143500,0$ & 195359,0 & 170464,4 \\
\hline Malaisie & 76,0 & 204,1 & - & $-25600,0$ & 294828,6 & 569709,2 \\
\hline Oman & - & - & - & - & 1260,9 & 136339,2 \\
\hline Ouzbékistan & 1422,8 & - & $-242,5$ & - & 3459,3 & 22538,7 \\
\hline Philippines & 2671,5 & 5464,5 & $-7009,2$ & $-166600,0$ & 117726,8 & 281059,0 \\
\hline Sri Lanka & 3235,1 & 1172,3 & - & $-7400,0$ & 55038,1 & 66109,5 \\
\hline
\end{tabular}


4.1. Relations avec les pays en développement, 2000, en milliers de francs (fin)

\begin{tabular}{|c|c|c|c|c|c|c|}
\hline Continent/pays & APD, aide bilat. & Aide priv. & GRE & IDE & Import. & Export. \\
\hline Syrie & 410,0 & 38,4 & - & - & 1917,8 & 72838,6 \\
\hline Thaillande & 1129,0 & 1019,6 & 402055,0 & 17300,0 & 605126,8 & 753004,6 \\
\hline $\begin{array}{l}\text { Zones sous admin. } \\
\text { palestinienne }\end{array}$ & 8916,2 & 987,6 & - & - & 76,8 & 3091,1 \\
\hline $\begin{array}{l}\text { Non ventilés } \\
\text { et projets régionaux }\end{array}$ & 12577,3 & 748,1 & - & $-19700,0$ & & \\
\hline Asie, non spécifié & 12577,3 & 748,1 & - & $-19700,0$ & & \\
\hline Océanie & 373,6 & 160,5 & - & $-6200,0$ & 348,9 & 2048,4 \\
\hline $\begin{array}{l}\text { Pays les moins avancés } \\
\text { (PMA) }\end{array}$ & 35,0 & 7,4 & - & - & 129,0 & 178,0 \\
\hline Samoa & - & 7,4 & - & - & 33,8 & 29,7 \\
\hline Vanuatu & 35,0 & - & - & - & 95,2 & 148,3 \\
\hline Pays à revenu moyen & 338,6 & 153,1 & - & - & 219,9 & 1870,4 \\
\hline Cook, Iles & - & - & - & - & 61,2 & 23,0 \\
\hline Fidji & - & 104,2 & - & - & 83,3 & 358,1 \\
\hline Micronésie & 50,0 & - & - & - & 40,7 & 54,5 \\
\hline Papouasie-Nlle-Guinée & 288,6 & 48,9 & - & - & 34,7 & 1434,8 \\
\hline $\begin{array}{l}\text { Non ventilés } \\
\text { et projets régionaux }\end{array}$ & - & - & - & $-6200,0$ & & \\
\hline Océanie, non ventilé & - & - & - & $-6200,0$ & & \\
\hline $\begin{array}{l}\text { Pays en développement } \\
\text { non spécifiés }\end{array}$ & 293539,0 & 23564,2 & - & - & & \\
\hline
\end{tabular}

Sources des tableaux 4.1. et 4.2.:

- Service statistique de la DDC, Berne pour les données sur l'APD, l'AP et la GRE.

- Aide suisse aux pays en développement et aux pays en transition 1998-2000, IUED, DDC, 2002.

- Banque nationale suisse pour les données sur l'investissement direct à l'étranger.

- Direction générale des douanes, Statistique annuelle du commerce extérieur de la Suisse 2000, statistiques annuelles 2000. Tome premier pp. 856-861 (pour les données sur les importations et les exportations). 


\subsection{Relations avec les pays en transition (aide publique à l'Est et aux PED plus avancés et flux privés), 2000, en milliers de francs}

$\begin{array}{llllll}\text { Continent/pays } & \text { APD, aide bilat. } & \text { Aide priv. } & \text { GRE } & \text { IDE } & \text { Import. }\end{array}$

\begin{tabular}{|c|c|c|c|c|c|c|}
\hline Total & 97758,4 & 13929,9 & 261785,3 & 10642500,0 & 9121654,7 & 9691835,8 \\
\hline $\begin{array}{l}\text { Pays en développement } \\
\text { plus avancés }\end{array}$ & 1776,9 & 3485,7 & $-13756,8$ & 9756300,0 & 3067409,8 & 5664700,1 \\
\hline Antilles Néerlandaises & - & 5,0 & - & 61100,0 & 1706,1 & 22376,6 \\
\hline Aruba & - & - & - & 61300,0 & 1,9 & 8401,2 \\
\hline Bahamas & - & - & - & 1010800,0 & 89003,4 & 78390,4 \\
\hline Bermudes & - & - & - & 6653400,0 & 27277,1 & 15373,0 \\
\hline Brunei & - & - & - & - & 3619,4 & 4694,2 \\
\hline Caimanes, Iles & - & - & - & 549000,0 & 2229,1 & 24225,4 \\
\hline Chypre & - & 25,0 & - & 481200,0 & 4781,7 & 78566,4 \\
\hline Corée & 74,2 & 453,3 & $-210,3$ & 137900,0 & 2669,2 & 9916,6 \\
\hline Emirats Arabes Unis & - & - & - & 70700,0 & 109740,1 & 679101,3 \\
\hline Gibraltar & - & - & - & 206900,0 & 41430,2 & 11121,3 \\
\hline Hong Kong, Chine & - & 107,4 & 5205,4 & $-86500,0$ & 894662,8 & 6261,7 \\
\hline Israël & 1673,0 & 2708,9 & $-18751,9$ & $-34100,0$ & 503339,8 & 1439639,1 \\
\hline Koweit & - & 10,4 & - & - & 6618,6 & 155090,9 \\
\hline Macao & - & - & - & - & 7501,4 & 2866,2 \\
\hline Nouvelle Calédonie & - & - & - & - & 169,7 & 1980,4 \\
\hline Singapour & - & - & - & 487800,0 & 284962,7 & 1599491,4 \\
\hline Taipei chinois (Taiwan) & 29,7 & 175,7 & - & 156800,0 & 1087696,6 & 1527204,0 \\
\hline \multicolumn{2}{|c|}{ PED plus avancés, non ventilé - } & - & - & - & & \\
\hline $\begin{array}{l}\text { Pays d'Europe centrale } \\
\text { et orientale, nouveaux } \\
\text { Etats indépendants }\end{array}$ & 95981,5 & 10444,2 & 275542,1 & 886200,0 & 6054244,9 & 4027135,7 \\
\hline Bélarus & 347,2 & 101,8 & - & - & 15599,8 & 24018,7 \\
\hline Bulgarie & 12563,8 & 962,1 & - & $-11700,0$ & 47604,1 & 143465,4 \\
\hline Estonie & 114,9 & - & $-2735,8$ & $-200,0$ & 47451,4 & 23313,6 \\
\hline Hongrie & 8354,3 & 550,1 & - & 55600,0 & 625330,7 & 656891,9 \\
\hline Lettonie & 1615,8 & 207,6 & $-1121,7$ & 14700,0 & 11672,7 & 65957,4 \\
\hline Lituanie & 86,1 & 65,7 & $-732,3$ & 4300,0 & 17243,6 & 63415,1 \\
\hline Pologne & 551,2 & 84,2 & 192682,2 & 264600,0 & 371389,6 & 1131969,0 \\
\hline Roumanie & 17148,5 & 5427,9 & 55867,3 & 346500,0 & 88755,1 & 252436,5 \\
\hline Russie & 17096,8 & 2193,3 & - & 35900,0 & 3843514,9 & 555661,1 \\
\hline Slovaque, Rép. & 2809,4 & 89,0 & 32071,8 & 12000,0 & 331023,3 & 216407,9 \\
\hline Tchèque, Rép. & 263,5 & 323,2 & $-489,5$ & 188400,0 & 604245,9 & 758398,5 \\
\hline Ukraine & 14817,6 & 379,8 & - & $-23900,0$ & 50413,8 & 135200,6 \\
\hline NEI, non ventilé & 2406,5 & - & - & - & & \\
\hline PECO, non ventilé & 9555,3 & 53,0 & - & - & & \\
\hline PECO/NEI, non ventilé & 8250,7 & 6,5 & - & - & & \\
\hline $\begin{array}{l}\text { Aide aux pays en transition } \\
\text { non ventilé }\end{array}$ & n, & - & - & - & & \\
\hline
\end{tabular}

Sources: voir tableau 4.1. 